\title{
CONTEÚDO LIPÍDICO E PERFIL DE ÁCIDOS GRAXOS DE CHLORELLA VULGARIS CULTIVADA EM ÁGUA PRODUZIDA
}

Bianca Bomfim Andrade1; Lucas Guimarães Cardoso1; Saulo Nascimento Mata1, Jamila Sueira de Jesus Silva ${ }^{2}$, Paulo Fernando de Almeida ${ }^{3}$, Janice Izabel Druzian ${ }^{4}$

${ }^{1}$ Instituto de Ciências da Saúde, Universidade Federal da Bahia; Salvador/Bahia; andrade.biancabomfim@gmail.com

${ }^{2}$ Faculdade de Farmácia, Universidade Federal da Bahia; Salvador/Bahia

${ }^{3}$ Laboratório de Biotecnologia e Ecologia de Microrganismos, Instituto de Ciências da Saúde, Universidade Federal da Bahia; Salvador/Bahia

${ }^{4}$ Departamento de Análises Bromatológicas, Faculdade de Farmácia, Universidade Federal da Bahia; Salvador/Bahia

Resumo: Estima-se que 80 a $90 \%$ da água fornecida às refinarias de petróleo e plantas petroquímicas seja descartada como água residual. A biodegradação é um importante processo de biorremediação da água produzida. Neste trabalho, a microalga Chlorella vulgaris foi cultivada em meio contendo diferentes concentrações de água produzida, avaliando a sua potencialidade para aplicação em biocombustíveis através do perfil de ácidos graxos das biomassas. Foram realizadas 3 condições de cultivo (30\%AP, 40\%AP e 50\%AP), sendo realizado a extração de lipídios, quantificação e identificação de ácidos graxos e cálculo teórico de biodiesel. O cultivo de C. vulgaris em meio contendo $40 \%$ de água produzida e $60 \%$ de meio BG- 11 possibilitou o maior acúmulo de lipídios e ácidos graxos.

Palavras-Chave: Microalga; Chlorella; Lipídios; Ácidos graxos; Água produzida.

\section{LIPID CONTENT AND FATTY ACIDS PROFILE OF CHLORELLA VULGARIS CULTIVATED IN PRODUCED WATER}

\begin{abstract}
: 80 to $90 \%$ of the water supplied to oil refineries and petrochemical plants is estimated to be disposed of as wastewater. Biodegradation is an important process of bioremediation of produced water. In this work, Chlorella vulgaris was cultivated in medium containing different concentrations of produced water, evaluating its potentiality for application in biofuels through the fatty acid profile of biomass. Three cultivation conditions were performed (30\% AP, $40 \%$ AP and 50\% AP), lipid extraction, quantification and identification of fatty acids and theoretical calculation of biodiesel. The cultivation of $C$. vulgaris in medium containing $40 \%$ of produced water and $60 \%$ of BG-11 medium allowed the largest accumulation of lipids and fatty acids.
\end{abstract}

Keywords: Microalgae; Chlorella; Lipids; Fatty acids; Produced water. 


\section{INTRODUÇÃO}

A água produzida consiste na água associada ao óleo durante o processo de extração do petróleo e pode ser resultante de dois processos: pela mistura do óleo do poço com a água do mar que se encontra ao redor deste ou pela injeção de água no campo de petróleo para levar o óleo profundo à superfície [1]. Estima-se que 80 a 90\% da água fornecida às refinarias de petróleo e plantas petroquímicas seja descartada como água residual, uma vez que a água não entra no produto final [2]. Devido a sua composição complexa (matéria em suspensão e contaminantes orgânicos, como alcanos e compostos aromáticos), é necessário tratá-la antes de seu descarte. Um processo típico de tratamento de efluentes petroquímicos inclui neutralização, floculação, filtração, clarificação e biodegradação [3].

A biodegradação é um importante processo de biorremediação da água produzida. Os microrganismos são utilizados para consumir o óleo dissolvido restante e outros poluentes orgânicos. Mais de 200 espécies de microrganismos podem ser utilizados neste processo de biorremediação, dentre eles algas e cianobactérias [2]. Chlorella vulgaris é uma microalga unicelular que possui grande potencial para a biorremediação de diferentes águas residuais e produção de bioenergia devido ao seu alto teor de óleo, condição de cultivo mixotrófico (utiliza-se fonte de carbono orgânico dissolvida no meio de cultivo), alta taxa de crescimento sob várias condições adversas e tolerância a altos níveis de metais pesados [4].

Neste trabalho, a microalga Chlorella vulgaris foi cultivada em meio contendo diferentes concentrações de água produzida, avaliando a sua potencialidade para aplicação em biocombustíveis através do perfil de ácidos graxos das biomassas.

\section{METODOLOGIA}

\section{1 Água residual da indústria petroquímica}

A água residual utilizada no estudo pertence a um poço de perfuração de petróleo

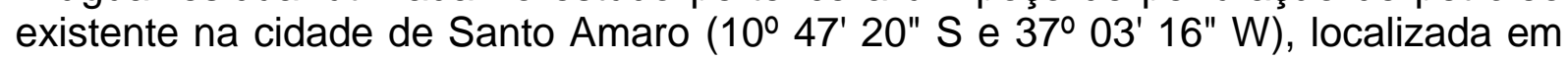
Sergipe. O referido poço é um dos pontos de perfuração da empresa Petrobras.

\subsection{Microalga e condições de cultivo}

A cepa da microalga Chlorella vulgaris foi obtida do Banco de Microalgas Iracema Nascimento, do Laboratório de Bioprospecção e Biotecnologia da Universidade Federal da Bahia (UFBA). C. vulgaris foi cultivada em 3 diferentes condições combinando o meio padrão BG-11 [5] e a água produzida nas seguintes proporções: $30 \%$ água produzida e 70\% meio BG-11 (AP30\%); 40\% água produzida e $60 \%$ meio BG-11 (AP40\%); e 50\% água produzida e 50\% meio BG-11 (AP50\%). Os experimentos foram realizados em triplicata e mantidos em fotobiorreator tipo Erlenmeyer, com volume de trabalho de $1 \mathrm{~L}$ e fotoperíodo 12 horas claro/escuro 
durante 7 dias. A iluminação foi fornecida com lâmpadas tubulares fluorescentes tipo luz diurna de $40 \mathrm{~W}$ que produziam uma iluminação de $41,6 \mu \mathrm{mol}$ fótons $\mathrm{m}^{-2} \mathrm{~s}^{-1}$ [6]. A água evaporada foi reabastecida a cada dia, antes da amostragem, com água destilada estéril. A aeração foi realizada através de injeção de ar. Os meios de cultivo foram esterilizados antes da montagem dos experimentos.

\subsection{Extração de lipídios e Composição de ácidos graxos}

O teor lipídico total das biomassas foi extraído e quantificado seguindo metologia descrita por Folch et al. (1957). [7]. A composição de ácidos graxos foi determinada através da transmetilação dos ácidos graxos com trifluoreto de boro hexano e identificação por cromatografia gasosa. Os ésteres metílicos de ácidos graxos (FAME) foram separados e identificados em um cromatógrafo a gás (Clarus 680; Perkin Elmer $\left.{ }^{\circledR}\right)$ com uma coluna DB-FFAP $(30 \mathrm{~m} \times 0,32 \mathrm{~mm} \times 0,25 \mathrm{~mm})$ equipado com um detector de ionização de chama. As injeções foram realizadas em duplicata para cada extração em um volume de $1 \mu \mathrm{L}$. Os tempos de retenção dos FAME foram comparados com uma mistura de padrões conhecidos (C4-C24, 18, 919-AMP; SigmaAldrich ${ }^{\circledR}$ ) para identificação destes. A quantificação dos ácidos graxos, expressa em concentração $\left(\mathrm{mg} \mathrm{g}^{-1}\right)$ porcentagem (\%) de lipídios, foi realizada pela adição do padrão interno tricosanoato de metila (C23:0; Sigma Aldrich®) [8]. As áreas dos picos foram determinadas através do software Clarus Chromatography workstation para normalizar a porcentagem de áreas de ácidos graxos totais [9].

\subsection{Análise estatística}

Os resultados foram analisados através do programa STATISTICA 10.0, utilizando análise de variância (ANOVA) seguida de teste de Tukey a 95,0\% de nível de confiança.

\section{RESULTADOS E DISCUSSÃO}

\subsection{Conteúdo lipídico}

A Figura 1 apresenta o conteúdo lipídico das biomassas cultivadas em $30 \%$, $40 \%$ e $50 \%$ de água produzida, respectivamente. Houve diferença estatística entre as porcentagens de lipídios das biomassas, sendo o maior conteúdo lipídico $(28,71 \%)$ observado em $40 \%$ AP e o menor em $50 \%$ AP $(9,16 \%)$. O maior acúmulo de lipídios nas biomassas com menor quantidade de água produzida em relação ao meio BG-11 pode ser devido à maior concentração de nutrientes essenciais para o metabolismo celular fornecidos pelo meio padrão, bem como pela menor concentração dos componentes da água produzida, que podem inibir a síntese lipídica na célula [10]. 


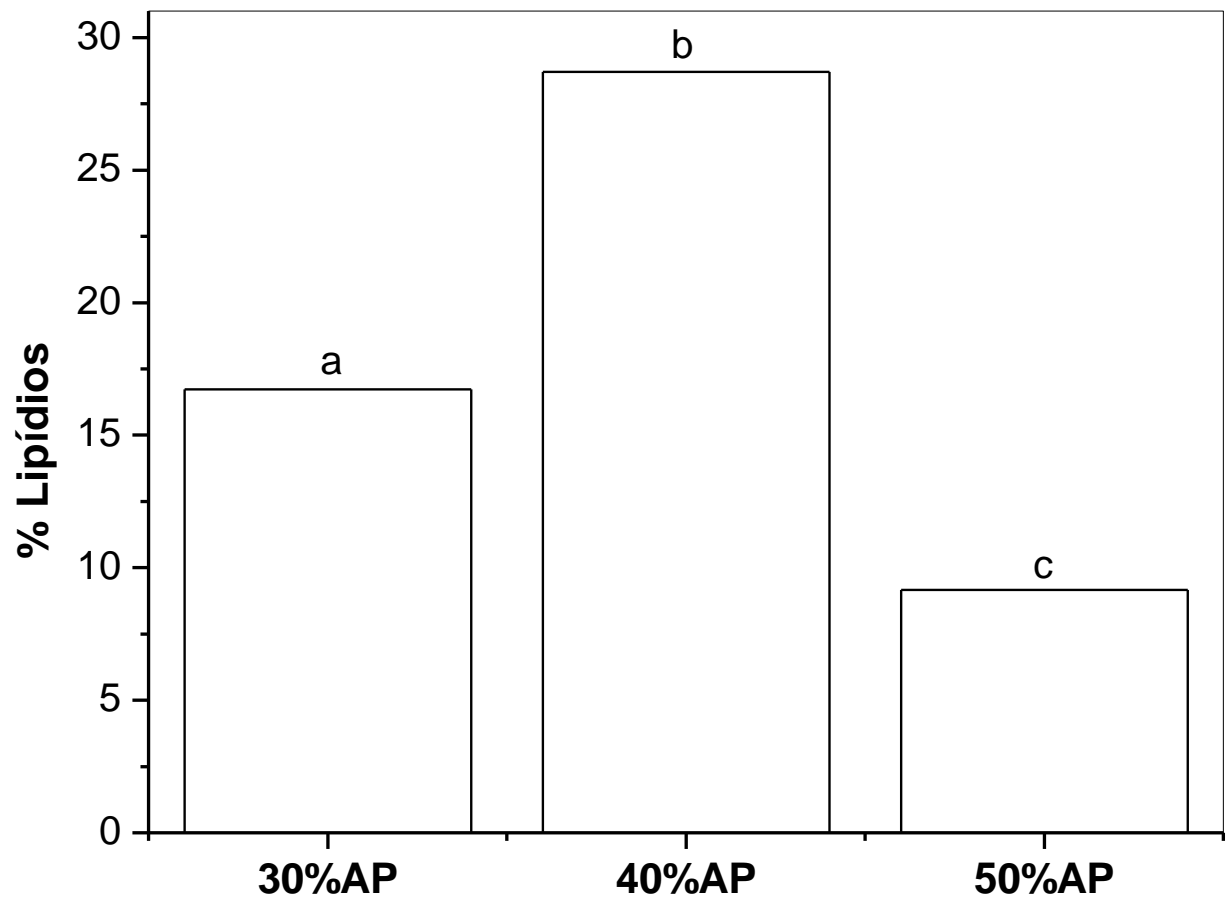

Figura 1. Conteúdo lipídico das biomassas de Chlorella vulgaris obtidas após cultivo em meio contendo $30 \%, 40 \%$ e $50 \%$ de água produzida.

Apesar do pequeno número de estudos envolvendo o cultivo de microalgas em água produzida, é possível comparar os resultados do presente trabalho com outros realizados em águas residuais da indústria petroquímica. O conteúdo lipídico de $C$. vulgaris cultivada em 40\%AP obtido neste trabalho foi superior ao da microalga Tribonema sp. cultivada em efluente do clarificador primário da indústria petroquímica [3]. Apesar de não. O cultivo de C. vulgaris na água produzida (AP40\%) permitiu maior acúmulo de lipídios que $C$. lobophora cultivada em água residual urbana e agrícola eutrófica (17,84\% e 20,40\%, respectivamente) [11].

\subsection{Composição de ácidos graxos}

De acordo com a Tabela 1, o total de ácidos graxos presentes nas biomassas de C. vulgaris cultivada em meio contendo $30 \%, 40 \%$ e $50 \%$ de água produzida foi estatisticamente diferente $(p<0,05)$, sendo a maior concentração de ácidos graxos encontrada na biomassa cultivada em $40 \%$ de água produzida $\left(840,64 \mathrm{mg}^{-1}\right)$ e a menor na biomassa cultivada em meio com $50 \%$ de água produzida $\left(465,37 \mathrm{mg} \mathrm{g}^{-1}\right)$. Do total de ácidos graxos encontrados na biomassa cultivada em $40 \%$ de água produzida, $79,41 \%$ corresponde a ácidos graxos monoinsaturados, principalmente C15:1 e C18:1 19 t. 
Tabela 1. Composição de ácidos graxos da biomassa de Chlorella vulgaris cultivada em água produzida $(30 \%, 40 \%$ e $50 \%)$.

\begin{tabular}{|c|c|c|c|c|c|c|}
\hline \multirow{2}{*}{ Ácido graxo } & \multicolumn{3}{|c|}{$\mathrm{mg} \mathrm{g}^{-1}$} & \multicolumn{3}{|c|}{$\%$} \\
\hline & $30 \% A P$ & $40 \% \mathrm{AP}$ & $50 \% A P$ & $30 \% A P$ & $40 \% A P$ & $50 \% A P$ \\
\hline $4: 0$ & ND & ND & 135,27 & ND & ND & 18,02 \\
\hline $6: 0$ & ND & ND & ND & ND & ND & ND \\
\hline 8:0 & ND & ND & ND & ND & ND & ND \\
\hline $10: 0$ & ND & 1,05 & 1,37 & 0,60 & 0,13 & 0,76 \\
\hline $11: 0$ & 4,58 & 5,82 & ND & 5,01 & 0,71 & ND \\
\hline $12: 0$ & 37,68 & 2,54 & ND & ND & 0,31 & ND \\
\hline $13: 0$ & ND & 3,04 & ND & ND & 0,37 & ND \\
\hline $14: 0$ & ND & ND & ND & 0,81 & ND & ND \\
\hline $14: 1 \omega 5$ & 6,08 & ND & ND & ND & ND & ND \\
\hline $15: 1$ & ND & 424,2 & 201,78 & 41,28 & 50,47 & 39,29 \\
\hline $16: 0$ & 310,22 & 12,57 & ND & 1,04 & 1,49 & ND \\
\hline $16: 1 \omega 7$ & 7,74 & 15,87 & ND & 2,46 & 1,93 & ND \\
\hline $17: 0$ & 18,46 & 13,6 & ND & 1,98 & 1,68 & ND \\
\hline $18: 0$ & 14,97 & 13,32 & ND & 1,94 & 1,59 & ND \\
\hline $18: 1 \omega 9 t$ & 14,61 & 227,88 & ND & 27,61 & 27,01 & ND \\
\hline $18: 2 \omega 6 c$ & 207,62 & 38,59 & 17,09 & 2,40 & 4,57 & 2,28 \\
\hline $18: 2 \omega 6 t$ & 18,12 & ND & 6,51 & ND & ND & 3,62 \\
\hline $18: 3 \omega 6$ & ND & 55,98 & ND & 6,57 & 6,67 & ND \\
\hline $18: 3 \omega 3$ & 49,37 & 26,18 & 72,38 & 5,50 & 3,08 & 24,94 \\
\hline $20: 3 \omega 6$ & 41,50 & ND & ND & ND & ND & ND \\
\hline $22: 2 \omega 6$ & ND & ND & 30,97 & 2,78 & ND & 11.09 \\
\hline Total & $751,87^{a}$ & $840,64^{b}$ & $465,37^{c}$ & 100,00 & 100,00 & 100,00 \\
\hline Saturados & $85,66^{a}$ & $51,94^{b}$ & $136,64^{c}$ & $11,38^{a}$ & $6,28^{\mathrm{b}}$ & $18,78^{c}$ \\
\hline Monoinsaturados & $536,30^{a}$ & $667,95^{\mathrm{b}}$ & $201,78^{\mathrm{c}}$ & $71,35^{a}$ & $79,41^{\mathrm{b}}$ & $39,29^{c}$ \\
\hline Poli-insaturados & $129,91^{\mathrm{a}}$ & $120,75^{\mathrm{b}}$ & $126,95^{\mathrm{c}}$ & $17,25^{\mathrm{a}}$ & $14,32^{\mathrm{b}}$ & $41,93^{c}$ \\
\hline
\end{tabular}

Letras iguais na mesma linha para $\mathrm{mg} \mathrm{g}^{-1} \mathrm{e} \%$ indicam que não há diferença significativa entre os experimentos a $95 \%$ de confiança.

O aumento dos ácidos graxos monoinsaturados pode ser devido a atividade da enzima fosfolipídio diacilglicerol aciltransferase (PDAT) numa via enzimática independente de acil-CoA, capaz de mediar a renovação dos lipídios da membrana e promover a síntese de triacilgliceróis (TAG) sob estresse. Além de funcionar como forma de armazenamento de carbono e energia, o TAG pode atuar na proteção celular, impedindo que as microalgas danifiquem a oxidação sob condições de estresse [12].

A água produzida contém uma mistura de compostos orgânicos e inorgânicos, sendo os constituintes principais: sais, sólidos dissolvidos totais, óleo, graxa, hidrocarbonetos poliaromáticos, benzeno, tolueno, etilbenzeno e xilenos, fenóis, ácidos orgânicos, compostos naturais orgânicos e inorgânicos, e aditivos químicos (biocidas e inibidores de corrosão) [1]. Desse modo, o cultivo em água produzida 
expõe a microalga a uma condição de limitação de nutrientes, dentre eles o nitrogênio, um dos principais nutrientes para a produção de biomassa algal.

As quantidades de C18:1 obtidas no presente estudo foram superiores às encontradas por Chi et al. (2019) [13] (17,21\%) na biomassa de Chlorella sp. cultivada em fotobiorreator de $250 \mathrm{~mL}$ em meio padrão. Zhu et al. (2015) [12] também observaram o aumento da porcentagem de C18:1 na biomassa de Chlorella zofingiensis cultivada sob limitação de nitrogênio $(31,96 \%)$ quando comparada à porcentagem do controle $(20,30 \%)$.

Os ácidos graxos monoinsaturados foram encontrados em maior quantidade também na biomassa cultivada em $30 \%$ de água produzida (536,30 mg g-1). Diferentemente, a biomassa cultivada em meio com $50 \%$ de água produzida apresentou maior quantidade de poli-insaturados, como C18:3w3 (24,94\%). Ördög et al. (2016) [14] também encontraram predominância do ácido $\alpha$-linoleico na biomassa de três cepas de Chlorella (Chlorella sp. MACC-438, Chlorella minutissima MACC-452 e Chlorella sp. MACC-728), porém em quantidades menores que no presente estudo $(9,0 \%, 10,8 \%$ e $9,0 \%$, respectivamente).

\section{CONCLUSÃO}

O cultivo de Chlorella vulgaris em meio contendo $40 \%$ de água produzida e $60 \%$ de meio BG-11 possibilitou o maior acúmulo de lipídios e ácidos graxos, os quais podem ser convertidos em biodiesel.

\section{Agradecimentos}

Os autores agradecem a Universidade Federal da Bahia (UFBA) e a FAPESB (Fundação de Amparo à Pesquisa do Estado da Bahia) - Brasil, projeto CNPq, MCTIC (Ministério da Ciência, Tecnologia, Inovações e Comunicações) - Brasil.

\section{REFERÊNCIAS}

${ }^{1}$ AL-GHOUTI, M. A.; AL-KAABI, M. A.; ASHFAQ, M. Y.; DA'NA, D. A. Produced water characteristics, treatment and reuse: A review. Journal of Water Process Engineering, v. 28, p. 222-239, 2019.

2 JAFARINEJAD, S., \& JIANG, S. C. Current technologies and future directions for treating petroleum refineries and petrochemical plants (PRPP) wastewaters. Journal of Environmental Chemical Engineering, v. 7, 2019.

${ }^{3}$ HUO, S.; CHEN, J.; ZHU, F.; ZOU, B.; CHEN, X.; BASHEER, S.; CUI, F.; QIAN, J. Filamentous microalgae Tribonema sp. cultivation in the anaerobic/oxic effluents of 
petrochemical wastewater for evaluating the efficiency of recycling and treatment. Biochemical Engineering Journal, v. 145, p. 27-32, 2019.

4 ZNAD, H.; AL KETIFE, A.M.D.; JUDD, S.; ALMOMANI, F.; VUTHALURU, H.B. Bioremediation and nutrient removal from wastewater by Chlorella vulgaris. Ecological Engineering, v. 110, p. 1-7, 2018.

${ }^{5}$ RIPPKA, R.; DERUELLES, J.; WATERBURY, J. W.; HERDMAN, M.; STANIER, R.G. Generic assignments, strain histories and properties of pure cultures of Cyanobacteria. Journal of General Microbiology, v. 111, p. 1-61, 1979.

${ }^{6}$ MORAIS, M.G.; COSTA, J.A.V. Carbon dioxide mitigation with Chlorella kessleri, Chlorella vulgaris, Scenedesmus obliquus and Spirulina sp. cultivated in flasks and vertical tubular photobioreactors. Biotechnology Letters, v. 29, p. 1349-1352, 2007.

7 FOLCH, J.; LEES, M.; SLOANE, G.H.S. A simple method for the isolation and purification of total lipides from animal tissues. Journal of Biological Chemistry, v. 226, p. 497-509, 1957.

8 JESUS, C.S.; UEBEL, L.S.; COSTA, S.S.; MIRANDA, A.L.; MORAIS, E.G.; MORAIS, M.G.; COSTA, J.A.V.; NUNES, I.L.; FERREIRA, E.S.; DRUZIAN, J.I. Outdoor pilotscale cultivation of Spirulina sp. LEB18 in different geographic locations for evaluating its growth and chemical composition. Bioresource Technology, v. 256, p. 86-94, 2018.

${ }^{9}$ NASCIMENTO, I. A.; MARQUES, S. S. I.; CABANELAS, I. T. D.; CARVALHO, G. C.; NASCIMENTO, M. A.; SOUZA, C. O.; DRUZIAN, J. I.; HUSSAIN, J.; LIAO, W. Microalgae Versus Land Crops as Feedstockk for Biodiesel: Productivity, Quality and Standard Compliance. Bioenergy Resource, v. 7, p. 1002-1013, 2014.

10 GAO, F.; YANG, H.; LI, C.; PENG, Y.; LU, M.; JIN, W.; BAO, J.; GUO, Y. Effect of organic carbon to nitrogen ratio in wastewater on growth, nutrient uptake and lipid accumulation of a mixotrophic microalgae Chlorella sp. Bioresource Technology, v. 282 , p. 118-124, 2019.

${ }^{11}$ KUMAR, K.S.; PRASANTHUMAR, S.; RAY, J.G. Biomass yield, oil productivity and fatty acid profile of Chlorella lobophora cultivated in diverse eutrophic wastewaters. Biocatalysis and Agricultural Biotechnology, v. 11, p. 338-344, 2017.

12 ZHU, S.; WANG, Y.; SHANG, C.; WANG, Z.; XU, J.; YUAN, Z. Characterization of lipid and fatty acids composition of Chlorella zofingiensis in response to nitrogen starvation. Journal of Bioscience and Bioengineering, v. 120, p. 205-209, 2015.

${ }^{13}$ CHI, N.T.L.; DUC, P.A.; MATHIMANI, T.; PUGAZHENDHI, A. Evaluating the potential of green alga Chlorella sp. for high biomass and lipid production in biodiesel viewpoint. Biocatalysis and Agricultural Biotechnology, v. 17, p. 184-188, 2019.

14 ÖRDÖG, V.; STIRK, W. A.; BÁLINT, P.; AREMU, A. O.; OKEM, A.; LOVÁSZ, C.; MOLNÁR, Z.; VAN STADEN, J. Effect of temperature and nitrogen concentration on lipid productivity and fatty acid composition in three Chlorella strains. Algal Research, v. 16, p. 141-149, 2016. 